\title{
Epicardial fat amount is associated with the magnitude of left ventricular remodeling in aortic stenosis
}

\author{
Augustin Coisne ${ }^{1,2,3,4,5} \cdot$ Sandro Ninni ${ }^{2,3,4,5} \cdot$ Staniel Ortmans ${ }^{1} \cdot$ Laurent Davin $^{6,7} \cdot$ Kevin Kasprzak $^{8}$. \\ Benjamin Longère $^{8}$. Claire Seunes ${ }^{1} \cdot$ Amandine Coppin $^{1} \cdot$ Stéphanie Mouton ${ }^{1} \cdot$ Hélène Ridon $^{1}$. Cedric Klein ${ }^{1}$. \\ Brieuc Noirot-Cosson ${ }^{1} \cdot$ Bart Staels $^{2,3,4,5,9} \cdot$ Patrizio Lancellotti ${ }^{6,7} \cdot$ David Montaigne ${ }^{1,2,3,4,5} \cdot$ François Pontana ${ }^{2,3,4,5,8}$
}

Received: 14 August 2018 / Accepted: 15 October 2018 / Published online: 17 October 2018

C) Springer Nature B.V. 2018

\begin{abstract}
Both genetic and environmental factors interact to control left ventricular (LV) remodeling in the context of aortic stenosis (AS). Epicardial adipose tissue (EAT) is a specific visceral adipose tissue with paracrine properties in close contact with the myocardium. We sought to assess determinants of EAT amount and its association with the magnitude and pattern of LV remodeling in patients suffering from severe AS. Between January 2014 and September 2017, we prospectively explored consecutive patients referred to our Heart Valve Center for SAVR presenting with severe AS and normal left ventricular ejection fraction (>50\%). Comprehensive transthoracic echocardiography (TTE) including assessment of LV remodeling and EAT amount were performed. 202 patients were included. EAT was significantly larger in elderly, diabetic and obese patients. EAT thickness was correlated positively with indexed LV mass in AS $\left(r^{2}=0.21 ; p<0.0001\right)$ as well as severe LV remodeling pattern. Importantly, this observation persisted after adjustment for other factors associated with LV remodeling $(\beta \pm \mathrm{SE}=1.74 \pm 0.34 ; \mathrm{p}<0.0001)$. Large amounts of EAT are positively and independently associated with more pronounced and severe LV remodeling in severe AS. Further exploration regarding the impact of functional properties of EAT on LV remodeling is required.
\end{abstract}

Keywords Aortic stenosis $\cdot$ Epicardial adipose tissue $\cdot$ Left ventricle remodeling $\cdot$ Hypertrophy $\cdot$ Obese

\section{Introduction}

In patients with aortic stenosis (AS), left ventricular hypertrophy $(\mathrm{LVH})$ develops as a response to chronic pressure overload. This ventricular remodeling is primarily adaptive, reducing wall stress and preserving systolic cardiac function.

Electronic supplementary material The online version of this article (https://doi.org/10.1007/s10554-018-1477-z) contains supplementary material, which is available to authorized users.

Augustin Coisne

augustin.coisne@chru-lille.fr

1 Department of Echocardiography and Cardiovascular Explorations - Heart Valve Clinic - Institut Cœur Poumon, CHU Lille, 59000 Lille, France

2 European Genomic Institute for Diabetes (E.G.I.D), FR 3508, 59000 Lille, France

3 Univ Lille, 59000 Lille, France

4 Inserm UMR 1011, 59000 Lille, France
Yet, LVH initiates a vicious circle leading to diastolic dysfunction, myocardial fibrosis, dilation, and finally heart failure [1]. Accordingly, LVH is associated with increased risk of cardiac events and poor prognosis in AS before and after surgical aortic valve replacement (SAVR) [2-4].

Genetic and environmental factors interact with the LV remodeling process in AS. Several studies reported these past few years that obesity, the metabolic syndrome, and type 2 diabetes are associated with more pronounced LVH [5-7].

5 Institut Pasteur de Lille, 59000 Lille, France

6 GIGA Cardiovascular Sciences, Departments of Cardiology, Heart Valve Clinic, University of Liège Hospital, Liège, Belgium

7 Gruppo Villa Maria Care and Research, Anthea Hospital, Bari, Italy

8 Department of Cardiovascular Radiology, CHU Lille, Lille, France

9 Department of Biology, CHU Lille, Lille, France 
However, the nature of the link between these metabolic disorders and cardiac remodeling is still poorly understood.

Visceral adipose tissue is a major source of adipokines and cytokines promoting insulin resistance and cardiovascular disorders in obese and diabetic patients [8]. Epicardial adipose tissue (EAT) is a visceral adipose tissue in close contact with the myocardium. Being located around the heart underneath the visceral pericardial layer, EAT is in direct contact with the epicardium [9]. EAT is thus a firstclass candidate to modulate the function of the myocardium and coronary vasculature [10].

Interestingly, EAT amount and its secretory/paracrine properties are altered in both obesity and type 2 diabetes $[8$, 11]. Moreover, visceral adipose tissue amount is independently associated with indexed left ventricular mass (LVMi) in patients with moderate AS [12].

Nevertheless, to our knowledge, there is no study on the specific association between EAT and ventricular remodeling in AS.

In the present study, we hypothesized that increased EAT amount is associated with poorer LV remodeling in AS.

\section{Methods}

\section{Study population and design}

From January 2014 to September 2017, we prospectively studied consecutive patients with severe AS referred to our Heart Valve Center (CHU Lille) for SAVR. Patients with reduced left ventricular ejection fraction $(<50 \%)$ were excluded to get a homogeneous population. Patients with another significant valvular disease, a medical history of previous cardiac surgery or congenital heart disease were also excluded.

The local ethics committee approved the protocol and patients gave informed consent.

\section{Cardiac imaging}

A comprehensive transthoracic echocardiography (TTE) was performed in all patients before after cardiac surgery using a commercially available ultrasound machine (Vivid E9, GE Healthcare, Little Chalfont, UK) and stored for off-line analysis (EchoPAC version 201.0.0; GE Vingmed Ultrasound, Horten, Norway). All measures were performed according to current guidelines, particularly for the assessment of AS, and LV structure and function [13].

\section{$\mathrm{LV}$ remodeling assessment}

LV mass was calculated using M-mode recordings according to the American Society of Echocardiography and the
European Association of Cardiovascular Imaging [13, 14]. LV mass was indexed to body height in the allometric power of 2.7 to get indexed LV mass (LVMi) [15]. LV hypertrophy $(\mathrm{LVH})$ was defined using the common cutoff values, i.e. $\mathrm{LVMi}>46.7 \mathrm{~g} / \mathrm{m}^{2.7}$ in women and $>49.2 \mathrm{~g} / \mathrm{m}^{2.7}$ in men.

Relative wall thickness (RWT) was calculated from TTE measures as the following ratio: $(2 \times \mathrm{LV}$ posterior wall thickness/LV end diastolic diameter).

Considering both LVMi and RWT, patients were classified as having one of the $4 \mathrm{LV}$ remodeling patterns using the following criteria: (1) normal pattern: absence of LVH and RWT <0.42; (2) concentric remodeling: absence of LVH and RWT $\geq 0.42$; (3) concentric hypertrophy: presence of LVH and RWT $\geq 0.42$; and (4) eccentric hypertrophy: presence of LVH and RWT $<0.42$.

\section{Epicardial adipose tissue assessment}

EAT was measured as the echo-free space between the outer wall of the myocardium and the visceral layer of the pericardium with TTE. EAT thickness was measured off-line with EchoPac (GE Healthcare), perpendicularly to the free wall of the right ventricle at end-systole in 3 cardiac cycles in the parasternal long-axis view as previously described [16]. Inter-observer reproducibility for this measurement was high with intraclass correlation coefficient of 0.93 IC [0.88; 0.96].

To further validate this method, EAT amounts were compared between TTE and cardiac magnetic resonance (CMR) in 84 patients explored for a LVH. The correlation between these two techniques was good in our center $(r=0.64$, $\mathrm{p}=0.0001)($ Supplemental Fig. 1).

\section{Statistical analysis}

Statistics were performed with MedCalc v16.4 (Olstead, Belgium). Continuous variables were tested for normality with Shapiro test, and are given as mean \pm SD. t-test and linear regression were performed for bi-variate analysis. Categorical variables are given as the number (percentage) of patients with the respective attribute and compared with Chi-square test.

Pearson correlation was used to explore the link between EAT thickness and LVMi. Chi-square for trend was used to assess relations between EAT amount and LV remodeling patterns, patients being divided on EAT thickness median. Multivariable linear regression analysis was performed to identify variables independently associated with LVMi. Variables with a p value $<0.10$ on univariable analysis were entered into the multivariable models.

A value of $p<0.05$ was considered statistically significant. Figures were drawn with GraphPad Prism version 5.0. 


\section{Results}

A total of 202 patients referred for a first SAVR were included. Patients' characteristics are summarized in Table 1 . Mean age was $69 \pm 10$ years. The population was made of $62 \%$ of male and a third had diabetes. $79 \%$ of them were overweight (43\%) or obese $(36 \%)$. Regarding medications, half of the patients was treated with betablockers or ACE inhibitors/ARBs.
EAT amount was larger in older $(\mathrm{p}=0.0063)$, hypertensive $(p=0.016)$ and diabetic patients $(p<0.0001)$ with higher BMI $(\mathrm{p}<0.0001)$ in bi-variate analysis (Table 2$)$. Conversely, gender was not associated with EAT thickness. After adjustment by multilinear regression, older age $(\mathrm{p}=0.01)$, higher BMI $(\mathrm{p}<0.0001)$ and diabetes mellitus $(p=0.008)$ remained significantly associated with larger EAT amount.

After splitting the population in half according to LVMi median, patients with higher LVMi had higher BMI
Table 1 Patients' characteristics $(\mathrm{n}=202)$

\begin{tabular}{|c|c|c|c|c|}
\hline & Total population $(n=202)$ & $\begin{array}{l}\text { Low LVMi } \\
(\mathrm{n}=102)\end{array}$ & $\begin{array}{l}\text { High LVMi } \\
(\mathrm{n}=100)\end{array}$ & $\mathrm{p}$ value \\
\hline \multicolumn{5}{|l|}{ Clinical data } \\
\hline Age (years) & $68.8 \pm 9.7$ & $67.9 \pm 10.4$ & $69.6 \pm 9.0$ & 0.22 \\
\hline Gender (male) n (\%) & $125(61.9)$ & $67(65.7)$ & $58(58.0)$ & 0.26 \\
\hline $\operatorname{BMI}\left(\mathrm{kg} / \mathrm{m}^{2}\right)$ & $28.9 \pm 4.9$ & $27.8 \pm 4.6$ & $29.9 \pm 4.9$ & 0.002 \\
\hline Normal n (\%) & $43(21.3)$ & $31(30.4)$ & $12(12.0)$ & 0.001 \\
\hline Overweight n (\%) & $87(43.1)$ & $44(43.1)$ & $43(43.0)$ & 0.98 \\
\hline Obese n $(\%)$ & $72(35.6)$ & $27(26.5)$ & $45(45.0)$ & 0.006 \\
\hline Hypertension n (\%) & $148(73.3)$ & $74(72.5)$ & $74(74.0)$ & 0.82 \\
\hline Diabetes mellitus n (\%) & $72(35.6)$ & $26(25.5)$ & $46(46.0)$ & 0.002 \\
\hline Smoking n (\%) & $55(27.2)$ & $25(24.5)$ & $30(30.0)$ & 0.38 \\
\hline NYHA class & $2.09 \pm 0.7$ & $2.09 \pm 0.7$ & $2.10 \pm 0.7$ & 0.97 \\
\hline Class I n (\%) & $5(2.5)$ & $1(1.0)$ & $4(4.0)$ & \\
\hline Class II n (\%) & $23(11.4)$ & $15(14.7)$ & $8(8.0)$ & \\
\hline Class III n (\%) & $122(60.4)$ & $60(58.9)$ & $62(62.0)$ & \\
\hline Class IV n (\%) & $52(25.7)$ & $26(25.5)$ & $26(26.0)$ & \\
\hline Euroscore $2(\%)$ & $1.50[1.05 ; 2.30]$ & $1.41[0.97 ; 2.20]$ & $1.53[1.13 ; 2.44]$ & 0.10 \\
\hline \multicolumn{5}{|l|}{ Treatments } \\
\hline Beta-blockers n (\%) & $71(35.1)$ & $33(32.4)$ & $38(38.0)$ & 0.40 \\
\hline ACE inhibitors/ARBs n (\%) & $100(49.5)$ & $50(49.0)$ & $50(50.0)$ & 0.89 \\
\hline Oral anti diabetic n $(\%)$ & $71(35.1)$ & $26(25.5)$ & $45(45.0)$ & 0.004 \\
\hline Insulin therapy $\mathrm{n}(\%)$ & $21(10.4)$ & $5(4.9)$ & $16(16.0)$ & 0.01 \\
\hline \multicolumn{5}{|l|}{ Biology } \\
\hline NT-pro-BNP (ng/l) & $396[180 ; 1052]$ & $367[158 ; 718]$ & $401[236 ; 1455]$ & 0.17 \\
\hline Creatinine (mg/l) & $9.0[8.0 ; 10.3]$ & $9.0[8.0 ; 10.1]$ & $9.0[7.7 ; 10.9]$ & 0.19 \\
\hline Cockroft et Gault (ml/min) & $79.5[67.0 ; 98.0]$ & $81.0[68.0 ; 99.3]$ & $78.0[63.5 ; 93.0]$ & 0.37 \\
\hline \multicolumn{5}{|l|}{ Cardiac imaging } \\
\hline Peak Ao velocity (m/s) & $4.40[4.14 ; 4.79]$ & $4.39[4.14 ; 4.60]$ & $4.50[4.15 ; 4.90]$ & 0.07 \\
\hline Mean Ao gradient (mmHg) & $50.5[42.5 ; 60.3]$ & $50.0[42.0 ; 55.1]$ & $52.0[43.9 ; 63.8]$ & 0.01 \\
\hline $\operatorname{iEOA}\left(\mathrm{cm}^{2} / \mathrm{m}^{2}\right)$ & $0.34[0.29 ; 0.42]$ & $0.34[0.30 ; 0.42]$ & $0.35[0.29 ; 0.42]$ & 0.95 \\
\hline $\operatorname{LVEF}(\%)$ & $61.3 \pm 9.3$ & $62.3 \pm 7.2$ & $60.2 \pm 35.7$ & 0.11 \\
\hline LA area $\left(\mathrm{cm}^{2}\right)$ & $24.5 \pm 8.0$ & $23.1 \pm 5.7$ & $25.9 \pm 9.7$ & 0.01 \\
\hline $\mathrm{ZVA}\left(\mathrm{mmHg} / \mathrm{ml} / \mathrm{m}^{2}\right)$ & $5.1[4.2 ; 6.1]$ & $5.1[4.3 ; 6.0]$ & $5.1[4.1 ; 6.5]$ & 0.59 \\
\hline $\operatorname{LVMi}\left(\mathrm{g} / \mathrm{m}^{2,7}\right)$ & $54.9 \pm 14.1$ & $44.0 \pm 6.9$ & $66.1 \pm 10.4$ & $<0.0001$ \\
\hline EAT thickness (mm) & $6.9 \pm 3.0$ & $5.7 \pm 2.4$ & $8.1 \pm 3.1$ & $<0.0001$ \\
\hline
\end{tabular}

Bold value represents $\mathrm{p}<0.05$ between Low LVMi and High LVMi

$A C E$ angiotensin-converting enzyme; Ao aortic; $A R B s$ Angiotensin II receptor blockers; $B M I$ body mass index; $E A T$ epicardial adipose tissue; $i E O A$ indexed effective orifice area; $L A$ left atrium; $L V E F$ left ventricular ejection fraction; $L V M i$ left ventricular mass index; NYHA New York Heart Association; ZVA valvuloarterial impedance 
Table 2 Determinants of epicardial adipose tissue thickness $(n=202)$

\begin{tabular}{|c|c|c|c|c|}
\hline & \multicolumn{2}{|l|}{ Univariable } & \multicolumn{2}{|c|}{ Multivariable } \\
\hline & $\beta \pm \mathrm{SE}$ & $\mathrm{p}$ & $\beta \pm \mathrm{SE}$ & $\mathrm{p}$ \\
\hline Gender (male) & $-0.69 \pm 0.43$ & 0.11 & & \\
\hline Age (years) & $0.06 \pm 0.02$ & 0.0063 & $0.05 \pm 0.02$ & 0.0103 \\
\hline BMI $\left(\mathrm{kg} / \mathrm{m}^{2}\right)$ & $0.33 \pm 0.04$ & $<0.0001$ & $0.25 \pm 0.04$ & $<0.0001$ \\
\hline Diabetes mellitus n (\%) & $2.01 \pm 0.42$ & $<0.0001$ & $1.07 \pm 0.39$ & 0.0083 \\
\hline Hypertension n (\%) & $1.17 \pm 0.46$ & 0.016 & - & - \\
\hline Mean aortic gradient (mmHg) & $-0.003 \pm 0.01$ & 0.84 & & \\
\hline Beta blockers & $-0.43 \pm 0.44$ & 0.33 & & \\
\hline ACE inhibitors/ARBs & $\mathbf{0 . 9 8} \pm 0.46$ & $\mathbf{0 . 0 3}$ & - & - \\
\hline
\end{tabular}

Bold value represents $\mathrm{p}<0.05$ between Low LVMi and High LVMi

p-value by t-test or linear regression in bivariate analysis. Regression coefficient, standard errors and $\mathrm{p}$-value from multivariate linear regression model constructed with all variables with $\mathrm{p}<0.10$ in bivariate analysis

$A C E$ angiotensin converting enzyme; $A R B s$ angiotensin II receptor blockers; $B M I$ body mass index

\section{Aortic stenosis $(\mathrm{n}=202)$}

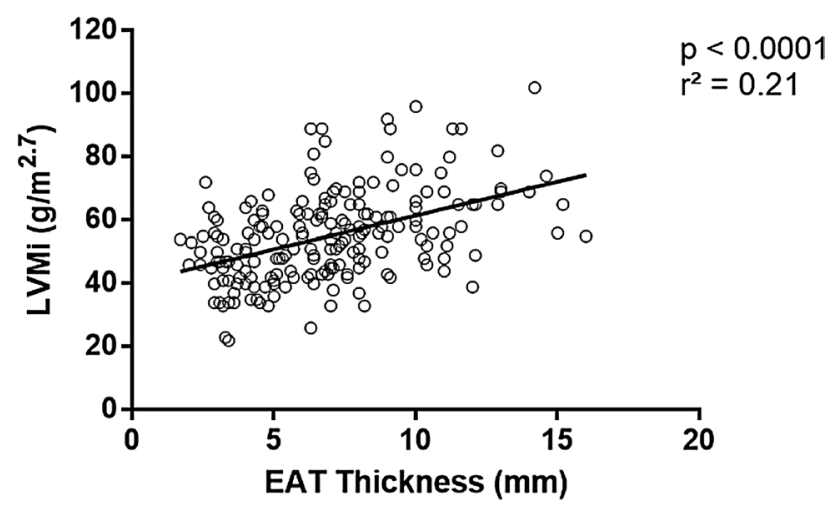

Fig. 1 Correlation between EAT amount and LV mass. $r^{2}$ and p-value by Pearson correlation and drawn line by linear regression

$\left(29.9 \pm 4.9\right.$ vs. $\left.27.8 \pm 4.6 \mathrm{~kg} / \mathrm{m}^{2}, \mathrm{p}=0.002\right)$, were more frequently diabetic (46 vs. $25 \%, 0.002$ ), with higher mean aortic gradient $(52.0$ [43.9; 63.8] vs. 50.0 [42.0; 55.1] $\mathrm{mmHg}, \mathrm{p}=0.01)$ and higher EAT thickness $(8.1 \pm 3.1$ vs; $5.7 \pm 2.4 \mathrm{~mm}, \mathrm{p}<0.001)$. This minimal difference between mean aortic gradient can be merely due to inter/ intra-observer variability and suggest that other factors can impact LV remodeling in AS.

We next explored the link between EAT amount and LVMi, an index of LV remodeling magnitude. Interestingly, LVMi correlated positively with EAT thickness $\left(\mathrm{r}^{2}=0.21, \mathrm{p}<0.0001\right)$ (Fig. 1). This association between EAT thickness and LVMi remained significant even after adjustment for other determinants of LVMi (Table 3). Overall the results were the same whatever the normalization of the LVM (Supplemental Table 1).
To further evaluate the link between LV remodeling and EAT, we explored whether EAT amount correlated with patterns of LV remodeling.

Very few patients $(20 ; 9.9 \%)$ displayed an absence of remodeling. 52 (25.7\%) patients had concentric LV remodeling, 104 (51.5\%) concentric LV hypertrophy and 26 (12.9\%) eccentric LV hypertrophy. Interestingly, the magnitude of LV remodeling was associated with EAT amount $(\mathrm{p}<0.0001$ by Chi-square for trend; Fig. 2).

Thus, in addition to the association between EAT quantity and LV mass, EAT amount was associated with a more severe LV remodeling pattern in AS.

\section{Discussion}

Exploring patients suffering from severe AS, we demonstrate that EAT amount is positively correlated with the extent of LVMi and with a more pathological remodeling pattern. Importantly, this observation persisted even after adjustment for other factors associated with LV remodeling.

\section{EAT, clinical metabolic disorders, and LV remodeling}

The human heart is coated with fat, which is in direct contact with the epicardium and is a source of free fatty acids, adipokines, and inflammatory cytokines [17]. Sharing a common microcirculation with the myocardium, EAT is believed to directly interact with cardiomyocytes in both physiology and pathology through paracrine signaling. Growing evidence supports this paradigm. On the one hand, EAT amount and quality have been shown to be altered by aging and metabolic disorders associated with high incidence of cardiovascular disease (CVD) [18, 19]. On the other hand, epidemiological studies demonstrated a 
Table 3 Determinants of indexed left ventricular mass normalized to height ${ }^{2.7}(\mathrm{n}=202)$

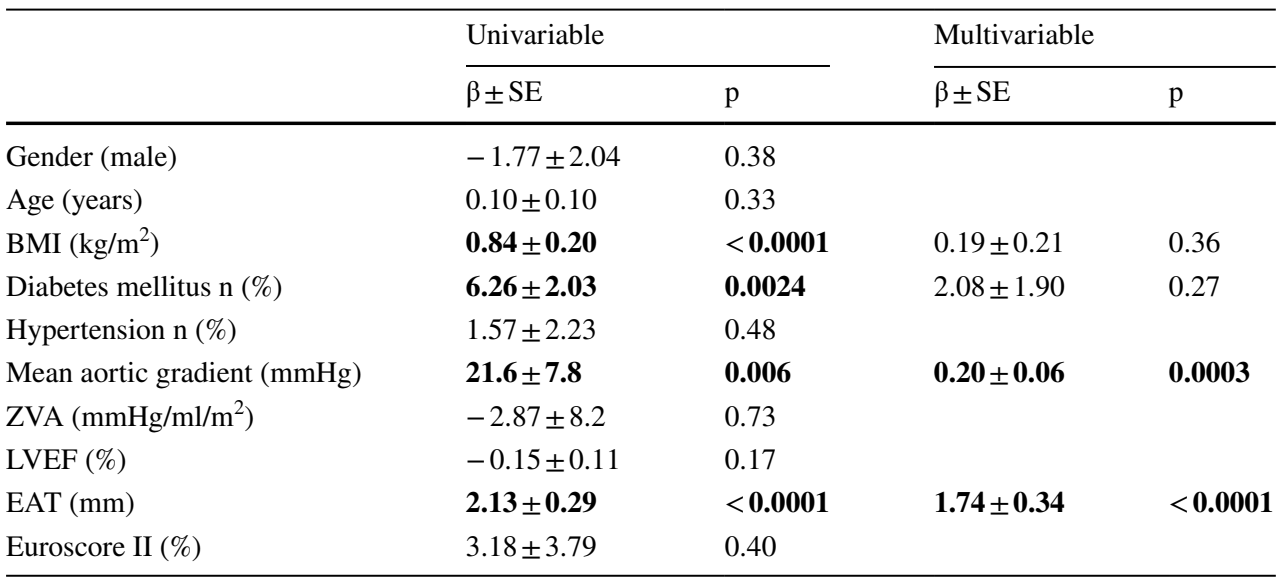

Bold value represents $p<0.05$ between Low LVMi and High LVMi

p-value by t-test or linear regression in bivariate analysis. Regression coefficient, standard errors and p-value from multivariate linear regression model constructed with all variables with $p<0.10$ in bivariate analysis

$B M I$ body mass index; EAT epicardial adipose tissue; $L V E F$ left ventricular ejection fraction; ZVA valvuloarterial impedance
Fig. 2 Patterns of LV remodeling according to EAT amount. Patients were divided in two groups according to EAT thickness median. p-value by Chisquare for trend

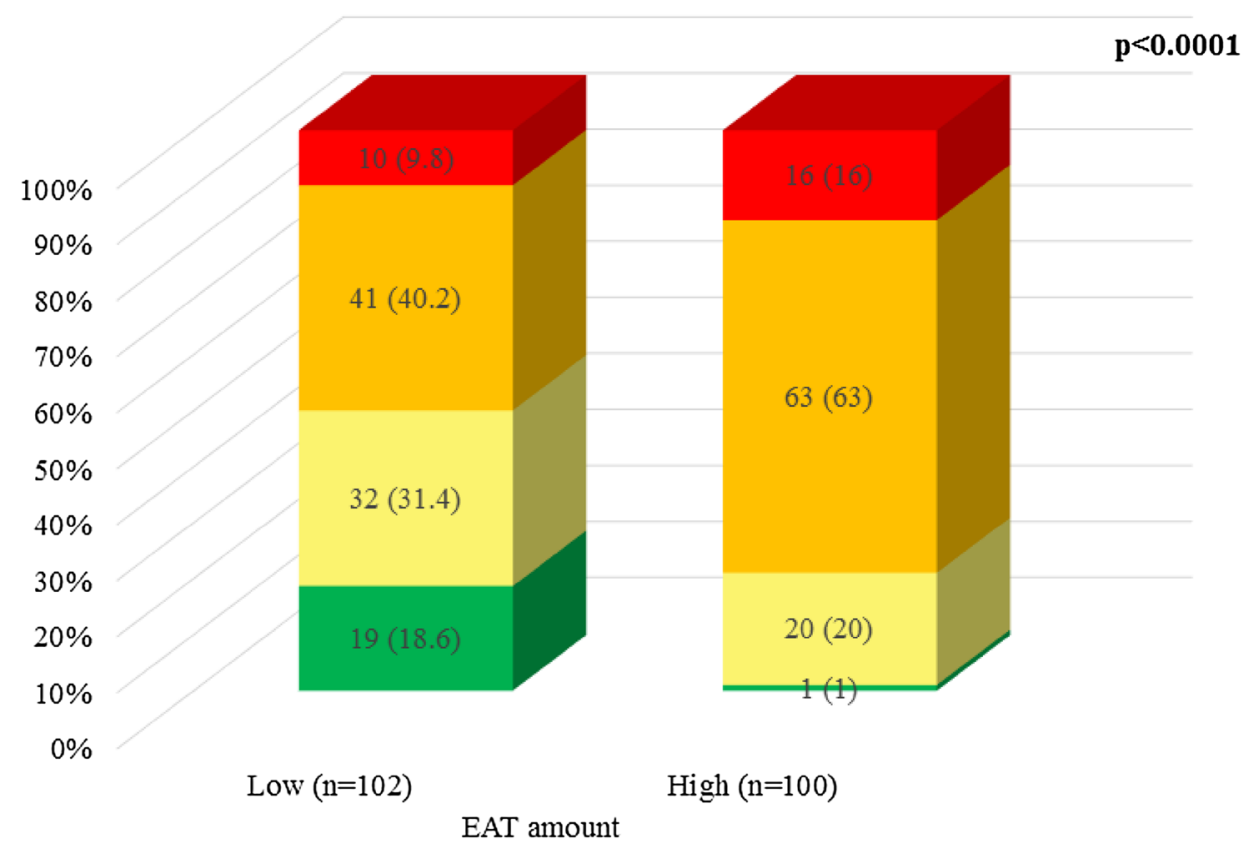

No Remodeling Concentric Remodeling $\square$ Concentric Hypertrophy $\square$ Eccentric Hypertrophy clear positive correlation between EAT volume and/or proinflammatory phenotype with CVD incidence and severity. Large EAT amounts, quantified by TTE [19], CT scan [20] or CMR [21], are consistently associated with severe coronary artery disease. Hirata et al. showed that infiltration of macrophages in EAT is enhanced in patients with coronary artery disease together with a shift of macrophage polarization toward a pro-inflammatory state, i.e. an increased M1/ M2 macrophage ratio [22]. These results suggested that EAT might influence atherogenesis in coronary arteries. In the same line, EAT amount and pro-inflammatory activity have been shown to be enhanced in atrial fibrillation patients [23].

Regarding EAT and cardiac remodeling, data from a few autopsy and echocardiographic studies reporting conflicting data are available. Coradi et al., exploring 117 human autopsy hearts, observed a constant ratio in each ventricle between EAT weight and muscle weight, even in presence of cardiac hypertrophy or features of ischemic cardiomyopathy 
[24]. The authors thus suggested that during the hypertrophic process, EAT and the ventricles presented a parallel and correlated increase in their masses. It is to be underlined that the etiology of cardiac hypertrophy was not reported in these 60 dead patients classified as having $<<$ hypertrophic hearts $>>$. Exploring 60 lean-to-obese healthy subjects, Iacobellis et al. showed a consistent positive correlation between EAT amount and LV mass [25]. Moreover, Capoulade et al. found that obesity and excess visceral adiposity (reflected by higher visceral adipose tissue/total adipose tissue ratio) were independently associated with LVH in 124 patients with AS [12]. Taken altogether, our results are consistent with Iacobellis and Capoulade findings and reinforce the concept that clinical conditions such as obesity, diabetes and aging may alter EAT, which in turn may impact the hypertrophic process in patients suffering from AS.

\section{Perspectives}

Taken together, our findings indicate that EAT amount is associated with the hypertrophic response in AS. Although the observational design of our study limits any conclusion regarding causality, evidence from basic science strongly supports a role for EAT in cardiac disorders observed in obesity and diabetes.

Yet, the precise mechanisms by which EAT and myocardium are associated in AS patients remain to be determined. Among the numerous adipokines and cytokines secreted by EAT, for only a few mechanistic data are available. Increases in TNFa, IL-10 or IL-6 or decreases in adiponectin signaling interfere with hypertrophic signaling pathways in rodent models and in vitro cardiomyocyte cultures [22]. Further exploration of the functional properties of EAT in the patients suffering from LV remodeling is required. This may open avenues for pharmacological strategies to modulate EAT activity and as such slow down the progression of LV remodeling in AS, specifically in those with obesity and diabetes.

\section{Limitations}

Our study has several limitations. First, we used a historical method (M-mode) that may have technical problems to calculate LVM compared to the more recent methods, including 3D. Second, EAT amount was estimated by measuring EAT thickness with TTE [16]. The linear measurement made with TTE on the right ventricular free wall cannot perfectly reflect the distribution and amount of EAT covering the LV. Nerlekar et al. recently found a poor correlation between EAT thickness measured by TTE and EAT area/ volume measured by using cardiac computed tomography (CT) $(r=0.29)$ [26]. Nevertheless, the amount of EAT was low in their patients evaluated with cardiac CT for suspected coronary artery disease. In opposition, we found a good correlation between EAT thickness by TTE and total EAT volume obtained by CMR in our center. Third, we did not report biological parameters such as insulin resistance index, serum triglyceride and cholesterol. This should be done in future studies to better characterize respective effects of obesity, the metabolic syndrome and type 2 diabetes on EAT.

\section{Conclusions}

Large EAT amounts are independently associated with more pronounced and severe LV remodeling AS. These new findings combined with previous bench studies argue for EAT being a potential actor of cardiac remodeling in the context of chronic pressure overload.

\section{Compliance with ethical standards}

Conflict of interest The authors declare that they have no conflict of interest.

\section{References}

1. Hill JA, Olson EN (2008) Cardiac plasticity. N Engl J Med 358(13): 1370-1380

2. Cioffi G, Faggiano P, Vizzardi E, Tarantini L, Cramariuc D, Gerdts E et al (2011) Prognostic effect of inappropriately high left ventricular mass in asymptomatic severe aortic stenosis. Heart Br Card Soc 97(4):301-307

3. Fuster RG, Argudo JAM, Albarova OG, Sos FH, López SC, Sorlí MJD et al (2003) Left ventricular mass index in aortic valve surgery: a new index for early valve replacement? Eur J Cardio Thorac Surg 23(5):696-702

4. Orsinelli DA, Aurigemma GP, Battista S, Krendel S, Gaasch WH (1993) Left ventricular hypertrophy and mortality after aortic valve replacement for aortic stenosis. A high risk subgroup identified by preoperative relative wall thickness. J Am Coll Cardiol 22(6):1679-1683

5. Capoulade R, Clavel M-A, Dumesnil JG, Chan KL, Teo KK, Tam JW et al (2013) Insulin resistance and LVH progression in patients with calcific aortic stenosis: a substudy of the ASTRONOMER trial. JACC Cardiovasc Imaging 6(2):165-1674

6. Lund BP, Gohlke-Bärwolf C, Cramariuc D, Rossebø AB, Rieck $\AA$ Á, Gerdts E (2010) Effect of obesity on left ventricular mass and systolic function in patients with asymptomatic aortic stenosis (a Simvastatin Ezetimibe in Aortic Stenosis [SEAS] Substudy). Am J Cardiol 105(10):1456-1460

7. Pagé A, Dumesnil JG, Clavel M-A, Chan KL, Teo KK, Tam JW et al (2010) Metabolic syndrome is associated with more pronounced impairment of left ventricle geometry and function in patients with calcific aortic stenosis: a substudy of the ASTRONOMER (Aortic Stenosis Progression Observation Measuring Effects of Rosuvastatin). J Am Coll Cardiol 55(17): 1867-1874

8. McNelis JC, Olefsky JM (2014) Macrophages, immunity, and metabolic disease. Immunity 41(1):36-48

9. Hassan M, Latif N, Yacoub M (2012) Adipose tissue: friend or foe? Nat Rev Cardiol 9(12):689-702 
10. Venteclef N, Guglielmi V, Balse E, Gaborit B, Cotillard A, Atassi $F$ et al (2015) Human epicardial adipose tissue induces fibrosis of the atrial myocardium through the secretion of adipo-fibrokines. Eur Heart J 36(13):795-805

11. Choe SS, Huh JY, Hwang IJ, Kim JI, Kim JB (2016) Adipose tissue remodeling: its role in energy metabolism and metabolic disorders. Front Endocrinol 7:30

12. Capoulade R, Larose E, Mathieu P, Clavel M-A, Dahou A, Arsenault $M$ et al (2014) Visceral adiposity and left ventricular mass and function in patients with aortic stenosis: the PROGRESSA study. Can J Cardiol 30(9):1080-1087

13. Lang RM, Badano LP, Mor-Avi V, Afilalo J, Armstrong A, Ernande L et al (2015 Jan) Recommendations for cardiac chamber quantification by echocardiography in adults: an update from the American Society of Echocardiography and the European Association of Cardiovascular Imaging. J Am Soc Echocardiogr 28(1):1.e14-39.e14

14. Galderisi M, Cosyns B, Edvardsen T, Cardim N, Delgado V, Di Salvo G et al (2017) Standardization of adult transthoracic echocardiography reporting in agreement with recent chamber quantification, diastolic function, and heart valve disease recommendations: an expert consensus document of the European Association of Cardiovascular Imaging. Eur Heart J Cardiovasc Imaging. 18(12): 1301-1310

15. Devereux RB, Alonso DR, Lutas EM, Gottlieb GJ, Campo E, Sachs I et al (1986) Echocardiographic assessment of left ventricular hypertrophy: comparison to necropsy findings. Am J Cardiol 57(6):450-458

16. Iacobellis G, Willens HJ (2009) Echocardiographic epicardial fat: a review of research and clinical applications. J Am Soc Echocardiogr 22(12):1311-1319

17. Mazurek T, Zhang L, Zalewski A, Mannion JD, Diehl JT, Arafat $\mathrm{H}$ et al (2003) Human epicardial adipose tissue is a source of inflammatory mediators. Circulation 108(20):2460-2466

18. Liang K-W, Tsai I-C, Lee W-J, Lee I-T, Lee W-L, Lin S-Y et al (2012) MRI measured epicardial adipose tissue thickness at the right AV groove differentiates inflammatory status in obese men with metabolic syndrome. Obesity 20(3):525-532

19. Eroglu S, Sade LE, Yildirir A, Bal U, Ozbicer S, Ozgul AS et al (2009) Epicardial adipose tissue thickness by echocardiography is a marker for the presence and severity of coronary artery disease. Nutr Metab Cardiovasc Dis NMCD 19(3):211-217

20. Vos AM de, Prokop M, Roos CJ, Meijs MFL, Schouw YT van der, Rutten A et al (2007) Peri-coronary epicardial adipose tissue is related to cardiovascular risk factors and coronary artery calcification in post-menopausal women. Eur Heart J 29(6): 777-783

21. Chen O, Sharma A, Ahmad I, Bourji N, Nestoiter K, Hua P et al (2015) Correlation between pericardial, mediastinal, and intrathoracic fat volumes with the presence and severity of coronary artery disease, metabolic syndrome, and cardiac risk factors. Eur Heart J 16 (1):37-46

22. Hirata Y, Tabata M, Kurobe H, Motoki T, Akaike M, Nishio C et al (2011) Coronary atherosclerosis is associated with macrophage polarization in epicardial adipose tissue. J Am Coll Cardiol 58(3):248-255

23. Al Chekakie MO, Welles CC, Metoyer R, Ibrahim A, Shapira AR, Cytron J et al (2010) Pericardial fat is independently associated with human atrial fibrillation. J Am Coll Cardiol 56(10):784-788

24. Corradi D, Maestri R, Callegari S, Pastori P, Goldoni M, Luong TV et al (2004) The ventricular epicardial fat is related to the myocardial mass in normal, ischemic and hypertrophic hearts. Cardiovasc Pathol 13(6):313-316

25. Iacobellis G, Ribaudo MC, Zappaterreno A, Iannucci CV, Leonetti F (2004 Oct) Relation between epicardial adipose tissue and left ventricular mass. Am J Cardiol 94(8):1084-1087

26. Nerlekar N, Baey Y-W, Brown AJ, Muthalaly RG, Dey D, Tamarappoo B et al (2018) Poor correlation, reproducibility, and agreement between volumetric vs. linear epicardial adipose tissue measurement: a 3D computed tomography vs. 2D echocardiography comparison. JACC Cardiovasc Imaging 11(7):1035-1036 